\title{
A novel statistical approach to detect differences in fat and protein test values among mid-infrared spectrophotometers ${ }^{1}$
}

\author{
Michael C. Adams and David M. Barbano² \\ Northeast Dairy Foods Research Center, Department of Food Science, Cornell University, Ithaca, NY 14853
}

\begin{abstract}
Our objective was to develop a statistical approach that could be used to determine whether a handler's fat, protein, or other solids mid-infrared (MIR) spectrophotometer test values were different, on average, from a milk regulatory laboratory's MIR test values when split-sampling test values are not available. To accomplish this objective, the Proc GLM procedure of SAS (SAS Institute Inc., Cary, NC) was used to develop a multiple linear regression model to evaluate 4 mo of MIR producer payment testing data (112 to 167 producers per month) from 2 different MIR instruments. For each of the 4 mo and each of the 2 components (fat or protein), the GLM model was Response $=$ Instrument + Producer + Date + 2-Way Interactions + 3-Way Interaction. Instrument was significant in determining fat and protein tests for 3 of the $4 \mathrm{mo}$, and Producer was significant in determining fat and protein tests for all 4 mo. This model was also used to establish fat and protein least significant differences (LSD) between instruments. Fat LSD between instruments ranged from 0.0108 to $0.0144 \%(\alpha=0.05)$ for the 4 mo studied, whereas protein LSD between instruments ranged from 0.0046 to $0.0085 \%(\alpha=0.05)$. In addition, regression analysis was used to determine the effects of component concentration and date of sampling on fat and protein differences between 2 MIR instruments. This statistical approach could be performed monthly to document a regulatory laboratory's verification that a given handler's instrument has obtained a different test result, on average, from that of the regulatory laboratory's and that an adjustment to producer payment may be required.
\end{abstract}

\footnotetext{
Received August 23, 2014.

Accepted February 2, 2015.

${ }^{1}$ Use of names, names of ingredients, and identification of specific models of equipment is for scientific clarity and does not constitute any endorsement of product by authors, Cornell University or the Northeast Dairy Foods Research Center.

${ }^{2}$ Corresponding author: dmb37@cornell.edu
}

Key words: statistics, mid-infrared, least significant difference, milk payment testing

\section{INTRODUCTION}

Typically, in the United States, thousands of raw milk samples are tested each day using mid-infrared (MIR) spectrophotometers to determine fat, protein, and other solids. These component measures are used in conjunction with delivery weights to determine payment to milk producers (i.e., dairy farmers) on a component weight basis (Barbano and Lynch, 2006). Because the trend is for fewer producers to provide larger volumes of milk per day (von Keyserlingk et al., 2013) to handlers (i.e., dairy plants), small errors in payment testing could amount to large amounts of money. The USDA Federal Milk Marketing Orders ensure accuracy of milk component testing by comparing MIR results from handlers' labs to the corresponding regulatory laboratory. Currently, this comparison can be made in 2 ways: through (1) split-sampling, or (2) statistical analysis of routine testing data for a group of producers (e.g., 100). Split-sampling is ideal because the same milk can be tested in both labs, thereby removing the uncertainty contributed by day-to-day variation and differences among samples. However, split-sampling can be difficult to carry out consistently from a logistical standpoint. It also requires twice as much milk and twice as many sample containers, making it a less sustainable practice. Split-sampling also has the disadvantage, from a verification perspective, that the handler knows these samples are being used to verify accuracy of testing. Statistical analysis of existing routine data could be used as an alternative to split-sampling to accomplish verification of handler testing accuracy when both labs are testing milks from the same group of producers during a month but on milks from different days if appropriate statistical procedures are used. In this approach, the normal day-to-day variation in milk composition for the same producer will present a challenge for accuracy, but this approach has the advantage that handlers can be checked at any time without prior knowledge that their performance is being evaluated. 
By building multiple linear regression models, least significant differences (LSD) can be determined at various confidence levels that take into account instrument, producer, and day-to-day variation. The statistical models can be constructed using only a few tests per producer per month (e.g., 3) and are not constrained to using identical duplicate milk samples for both MIR analyses on the same day. This method offers flexibility in sample collection and would be robust to the logistical problems associated with split-sampling of large groups of individual producers. In addition, random selection of milk samples within a given period (e.g., a 10-d period) for statistical analysis would prevent handlers from knowing when their results were being compared with the regulatory laboratory's results.

Our objective was to develop a statistical approach that could be used to determine whether a handler's fat, protein, or other solids MIR test values were different, on average, from a regulatory laboratory's MIR test values when split-sampling is not practical. To accomplish this objective, we developed a multiple linear regression model to evaluate MIR producer payment testing data; then, fat and protein LSD between instruments using this model were calculated. In addition, regression analysis was used to determine the effects of component concentration and date of sampling on fat and protein differences between 2 MIR instruments. This statistical approach could be performed monthly to document a regulatory laboratory's verification that a given handler's instrument has obtained a different test result, on average, from that of the regulatory laboratory's and that adjustments to producer payment and the handler's MIR instrument may be required.

\section{MATERIALS AND METHODS}

\section{Data Acquisition and Organization}

Fat and protein testing data from 2 anonymous MIR instruments (arbitrarily designated "Instrument 1" and "Instrument 2") used to test producer raw milks collected from a common set of northeast UF milk producers were supplied by USDA Federal Milk Marketing Order 1 (Albany, NY) for the months of September 2010, December 2010, March 2011, and July 2011. Within these months, payment testing data from 112, 149, 150, and 167 producers were analyzed, respectively.

Eight data sets $(2$ components: fat and protein $\times$ 4 mo: September, December, March, and July) were created and independently analyzed using the methods described below. To populate each of these data sets, 3 test values were randomly chosen from each instrument for each producer within each month. These 3 test val- ues represented milk samples collected during each of 3 periods within a month: the beginning of the month, the middle of the month, and the end of the month. The beginning, middle, and end of the month periods encompassed d 1 to 10 of the month, d 11 to 20 of the month, and d 21 to the end of the month, respectively. The dates on which milk samples were collected were not the same for the 2 instruments for a given producer during a given period. This choice was made to permit flexibility when selecting samples for comparison of results from 2 laboratories. The average difference in sample date between 2 instruments' test values within a common 10-d period for a common producer was 2.2 $\mathrm{d}$ for the 8 data sets studied. The median difference was $2 \mathrm{~d}$ for all 8 data sets.

\section{Statistical Model Development and Analyses}

Multiple Linear Regression Model. A multiple linear regression model was constructed using SAS software (version 9.2, SAS Institute Inc., Cary, NC). The following general linear model (PROC GLM) was used:

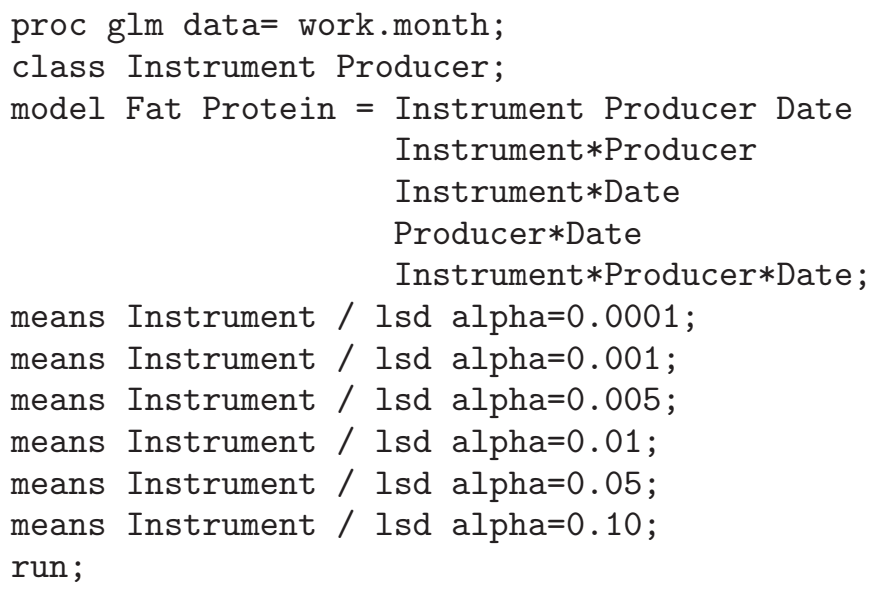

The ANOVA model included factors to account for variation contributed by the 2 MIR instruments used (Instrument), the producers from which raw milk samples were collected (Producer), the date of milk sampling (Date), and the interactions among these factors. Instrument and Producer were treated as categorical factors and Date was treated as a continuous factor. Furthermore, Date was mean-centered before model development to reduce the likelihood of multicollinearity and to improve the precision of its parameter estimates (Glantz and Slinker, 2001). Mean centering was performed by subtracting the average of all sample dates within a month from each sample date. For this application, the only factor levels of interest for a given analysis would be those instruments and producers be- 
ing used in the model. Consequently, all factors were considered fixed, not random.

The assumptions for multiple linear regression dictate that (1) the plot of predicted test values versus actual tests values should be linear, (2) the model residuals should be independent of one another and normally distributed, and (3) variance should be constant throughout the range of test values. All assumptions were verified for all data sets studied as described by Lawson (2010).

Least Significant Differences. The SAS model above was used to calculate Instrument LSD values for $\alpha$ levels $0.0001,0.001,0.005,0.01,0.05$, and 0.10 according to Equation [1], where $t$ is the $t$-statistic, $n$ is the number of test values for each instrument in a month, and $M S E$ is the mean squared error of the multiple linear regression model:

$$
L S D=t_{\alpha / 2, n-2} \sqrt{\frac{2 \times M S E}{n}} .
$$

Instrument Test-Value Difference Plots. For each producer within a given 10-d period (i.e., beginning, middle, or end of the month), Instrument 2 fat or protein values were subtracted from Instrument 1 fat or protein values and these differences were plotted as a function of their respective Instrument 1 values using Microsoft Excel (Redmond, WA). Lines of best fit were constructed for these plots using the Regression data function. The null hypothesis, $\mathrm{H}_{0}$ : the slope of the line of best fit $=0$, was tested for each line. In addition, coefficients of determination $\left(\mathrm{R}^{2}\right)$ were calculated to establish the degree of correlation between test value differences and component concentration. Test value differences were also plotted as a function of the date of the month (from Instrument 1 test values), and the same procedure was performed to examine the correla- tions between test value differences and the date of sampling (i.e., differences as a function of time).

\section{RESULTS AND DISCUSSION}

\section{Statistical Model ANOVA}

The degrees of freedom, type III sums of squares (SS), and $P$-values for the multiple linear regression ANOVA models for fat and protein test values are given in Tables 1 and 2, respectively. To determine whether the 2 instruments were performing differently, the most important model factor to examine was Instrument. For the months of September, December, and March, Instrument was significant $(P<0.05)$ in determining the fat test (Table 1). For the months of September, March, and July, Instrument was significant $(P<0.001)$ in determining the protein test (Table 2). These findings indicate that after accounting for variation contributed by Producer, Date, and their interactions, the MIR instrument used to measure fat and protein still influenced $(P<0.05)$ test results in 6 of the 8 models. If a $t$-test were used directly on the test results instead of ANOVA, it would be more difficult to establish differences between instruments because variation attributable to the producers and date of sampling would not be filtered out before analysis. Because Producer was always important in determining test values and Date was important in 5 of the 8 models (Tables 1 and 2), Instrument $P$-values derived from an unpaired, equal variance $t$-test (Instrument only) were always greater than those found using ANOVA (Table 3). This implies that multiple linear regression-based ANOVA is more sensitive at identifying differences in test values among instruments than using a $t$-test.

The interaction between Instrument and Producer was never significant $(P>0.05)$ in determining the fat

Table 1. Analysis of variance with degrees of freedom, type III sums of squares (SS), and $P$-values for the predictive variables of fat test values for 2 mid-infrared spectrophotometers for 4 mo during 2010 and 2011

\begin{tabular}{|c|c|c|c|c|c|c|c|c|c|c|c|c|}
\hline Item & \multicolumn{3}{|c|}{ September 2010} & \multicolumn{3}{|c|}{ December 2010} & \multicolumn{3}{|c|}{ March 2011} & \multicolumn{3}{|c|}{ July 2011} \\
\hline Instrument (I) & 1 & 0.06 & $* *$ & 1 & 0.02 & $*$ & 1 & 0.06 & $* *$ & 1 & 0.03 & 0.15 \\
\hline Date (D) & 1 & 0.20 & $* * *$ & 1 & 0.03 & $*$ & 1 & 0.04 & $*$ & 1 & 0.05 & 0.05 \\
\hline $\mathrm{I} \times \mathrm{P}$ & 111 & 0.40 & 1.00 & 148 & 0.52 & 1.00 & 149 & 1.20 & 0.48 & 166 & 1.70 & 0.98 \\
\hline $\mathrm{I} \times \mathrm{D}$ & 1 & 0.00 & 0.80 & 1 & 0.01 & 0.22 & 1 & 0.01 & 0.17 & 1 & 0.00 & 0.69 \\
\hline Error & 224 & 1.7 & - & 298 & 2.0 & - & 300 & 2.4 & - & 334 & 4.5 & - \\
\hline Total & 671 & 35.0 & - & 893 & 49.5 & - & 899 & 54.9 & - & 1,001 & 55.4 & - \\
\hline $\mathrm{R}^{2}$ & & 0.952 & & & 0.959 & & & 0.956 & & & 0.919 & \\
\hline Model $P$-value & & $<0.0001$ & & & $<0.0001$ & & & $<0.0001$ & & & $<0.0001$ & \\
\hline
\end{tabular}

${ }^{*} P<0.05 ;{ }^{* *} P<0.01 ;{ }^{* * *} P<0.001$. 
Table 2. Analysis of variance with degrees of freedom, type III sums of squares (SS), and $P$-values for the predictive variables of protein test values for 2 mid-infrared spectrophotometers for 4 mo during 2010 and 2011

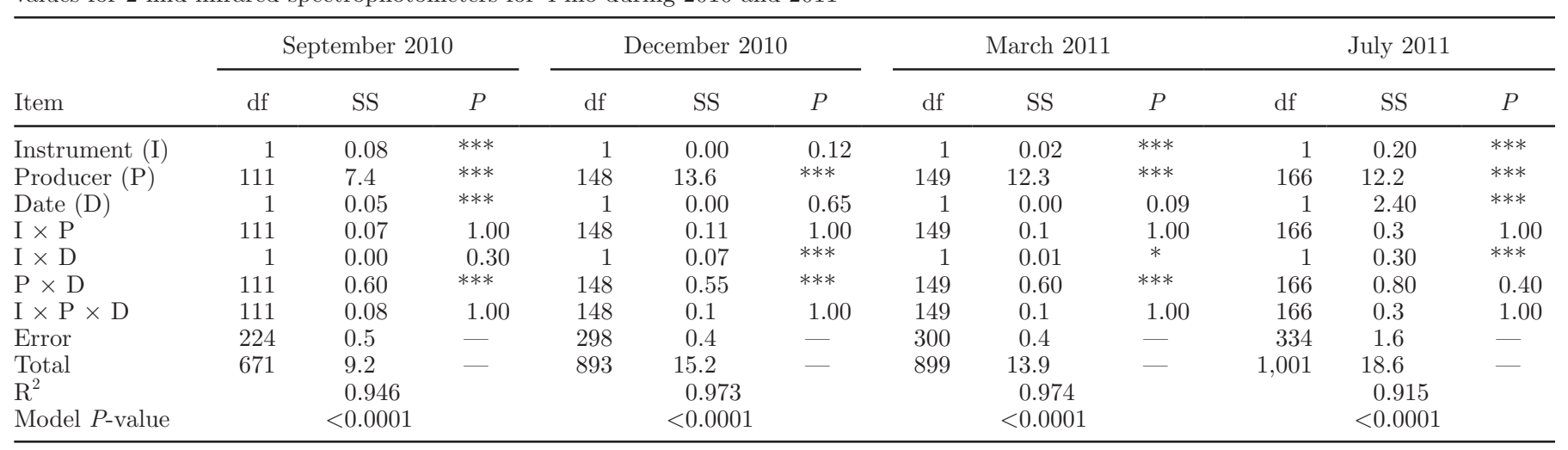

${ }^{*} P<0.05 ;{ }^{* *} P<0.01 ;{ }^{* * *} P<0.001$.

or protein tests in these data sets. A contrary result would have indicated that the 2 MIR instruments were producing different test results that were dependent on the individual producers being analyzed. Previous work has shown that FA chain length and degree of unsaturation can influence MIR fat tests (Kaylegian et al., 2009a,b). If several producers were providing milks with different FA profiles that caused them to be analyzed differently than the rest of the population by one instrument, there would be reduced power in determining differences among instruments. Therefore, it is important that the possible cause of this interaction be considered if it is significant. In the case of a significant interaction $(P<0.05)$, the analyst should interpret the results of the statistical test based on the significant interaction, and not the main effect.

During the months of December, March, and July, the interaction between Instrument and Date was significant $(P<0.05)$ in determining the protein test (Table 2$)$. This indicated that the protein test differences between instruments for these months were influenced by the date of sampling. Fat and protein test values varied in several of the months depending on the producer supplying the milk, as evidenced by significant interactions $(P<0.001)$ between Producer and Date in 7 of the 8 models (Tables 1 and 2). The 3 -way interaction was not important $(P>0.05)$ in determining the fat or protein tests in any of the models examined (Tables 1 and 2).

\section{LSD}

The Instrument LSD values derived from the ANOVA model for fat tests ranged from 0.0108 to $0.0144 \%$ ( $\alpha$ $=0.05$ ) for the 4 mo studied (Figure 1). The average LSD value for fat tests between instruments for the 4 mo was $0.0126 \%(\alpha=0.05)$. Decreasing the $\alpha$ level (i.e., increasing the confidence level) increased both fat and protein LSD values (Figures 1 and 2). At a common $\alpha$ level, Instrument LSD values for protein tests were always lower than those derived for fat tests. This was expected because protein content varies to a lesser degree among milkings than fat content (Gilbert et al., 1973). The Instrument LSD values for protein tests ranged from 0.0046 to $0.0085 \%(\alpha=0.05)$ for the $4 \mathrm{mo}$ studied (Figure 2). The average LSD value for protein tests between instruments for the 4 mo was $0.0063 \%$ ( $\alpha$ $=0.05$ ). Because lower LSD values are associated with increased confidence, a regulatory laboratory would have to choose the level of confidence that they are comfortable with before making a claim of difference between the handler and regulatory laboratory.

The accuracy of LSD can be improved by removing nonsignificant $(P>0.05)$ factors from the multiple linear regression model before estimation of the LSD. Removing factors from a model (i.e., making a reduced model) will simultaneously increase the degrees of freedom of the error term and increase the error type III

Table 3. $P$-values for the significance of the predictive factor Instrument on fat and protein test values determined using ANOVA or two-tailed, equal variance $t$-tests for 4 mo during 2010 and 2011

\begin{tabular}{lcccc}
\hline Item & September 2010 & December 2010 & March 2011 & July 2011 \\
\hline Fat ANOVA & 0.005 & 0.041 & 0.007 & 0.146 \\
Fat $t$-test & 0.226 & 0.411 & 0.331 & 0.373 \\
Protein ANOVA & $<0.0001$ & 0.118 & $<0.0001$ & $<0.0001$ \\
Protein $t$-test & 0.011 & 0.678 & 0.195 & 0.002 \\
\hline
\end{tabular}




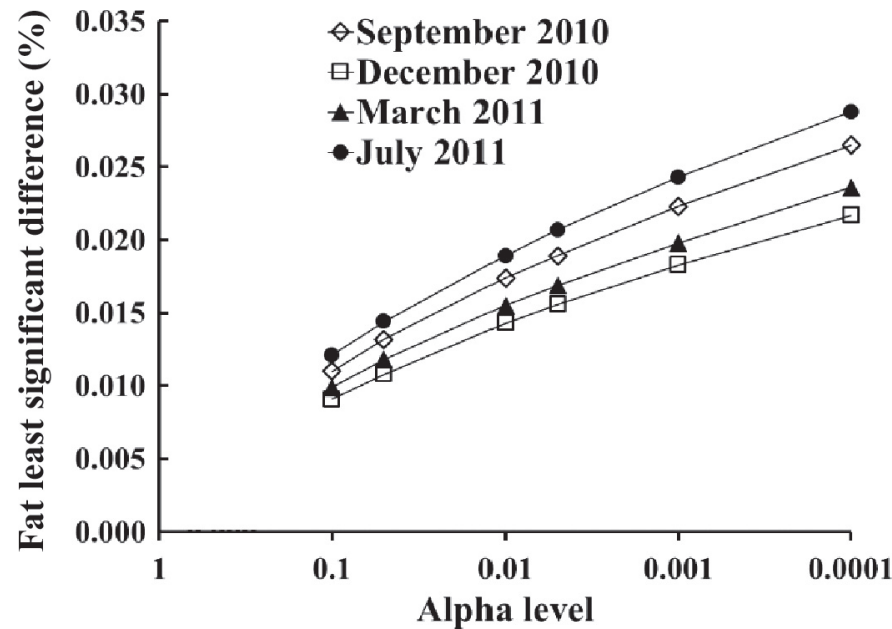

Figure 1. Least significant differences between 2 mid-infrared spectrophotometers' average monthly fat tests for the months of September 2010, December 2010, March 2011, and July 2011 as functions of confidence level.

SS. If the factors that were removed contributed little to the model's predictive ability, the increase in the error SS would likely be small relative to the increase in the degrees of freedom for the error term, causing the mean squared error to decrease. This would cause LSD values to decrease (Equation [1]). For the data sets examined, LSD values decreased between 8 and $20 \%$ when nonsignificant factors $(P>0.05)$ were removed from the model (data not shown).

\section{Instrument Difference Plots}

Plots of fat and protein test differences among instruments as they correlated to Instrument 1 fat or protein concentrations and date of the month during July are illustrated in Figure 3. Tables 4 and 5 provide details regarding the lines of best fit when these differences were correlated to Instrument 1 component concentration and date of the month, respectively, for each month studied.

Effect of Instrument 1 Concentration on Instrument Differences. If a relationship existed between the difference in instrument test values and one of the instruments' test values, individual producers who provide milks with low or high concentrations of fat or protein may be underpaid or overpaid relative to the average of all producers even though, on average, no significant difference may exist between instruments (i.e., the mean of the 2 instruments agree, but there is a significant difference in slope between the instruments). Scatterplots such as those in Figure 3 could be used to quickly detect these systematic biases at low and high concentrations. Slopes of the lines of best fit for these

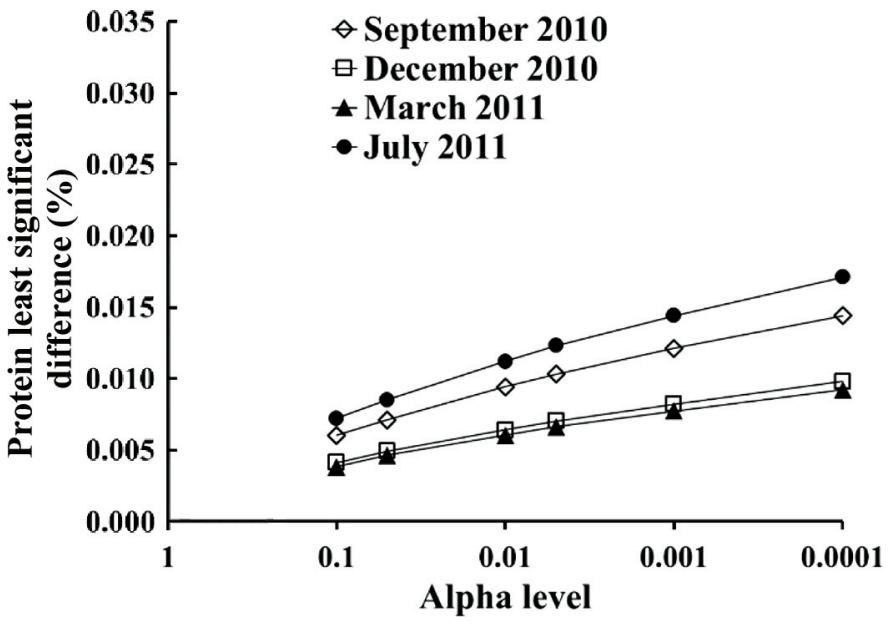

Figure 2. Least significant differences between 2 mid-infrared spectrophotometers' average monthly protein tests for the months of September 2010, December 2010, March 2011, and July 2011 as functions of confidence level.

plots were different $(P<0.01)$ from zero in 6 of the 8 data sets examined (Table 4 ). This indicated that there was a trend in instrument differences across the range of fat or protein concentrations. However, these trends are unlikely to be important because no strong correlations $\left(\mathrm{R}^{2}<0.10\right)$ were detected during the 4 mo studied (Table 4). In correlation analyses, the $t$-statistic used to determine whether a line is significantly different from zero is increased (i.e., made more significant) as the number of observations increases (Ott and Longnecker, 2004). Therefore, large sample sizes such as those in these data sets (e.g., >100 producers) increase the likelihood of determining a slope to be different from zero, regardless of correlation. This finding was consistent with the ANOVA. Because each point in these scatterplots represents a difference between instruments concerning a single producer, it can be reasoned that a strong correlation in this plot should also manifest itself as a significant Instrument $\times$ Producer interaction in the ANOVA model. As expected, no significant $(P$ $>0.05$ ) interactions between Instrument and Producer were identified in any of the 8 ANOVA models (Tables 1 and 2).

Effect of Date of the Month on Instrument Differences. No strong correlations were observed between differences in fat tests and date of the month $\left(\mathrm{R}^{2}\right.$ $<0.01$ ) for any of the 4 mo studied (Table 5). In addition, none of these best-fit lines exhibited slopes that were significantly different from zero $(P>0.05$; Table $5)$. Consequently, the differences in fat tests between the 2 instruments were not found to be correlated to the date of sampling. This was confirmed with the fat test ANOVA, as the interaction between Instrument 
Table 4. Linear regression parameters describing the effects of fat and protein concentrations on the differences in fat and protein test values, respectively, between 2 mid-infrared spectrophotometers for 4 mo during 2010 and 2011

\begin{tabular}{lccrr}
\hline Item & September 2010 & December 2010 & March 2011 & July 2011 \\
\hline Fat slope & 0.0848 & 0.0670 & 0.0647 & 0.1513 \\
Fat $P$-value & $<0.0001$ & 0.0003 & 0.0036 & $<0.0001$ \\
Fat $^{2}$ & 0.0509 & 0.0297 & 0.0187 & 0.0743 \\
Protein slope $_{\text {Protein } P \text {-value }}^{1}$ & 0.0557 & 0.0202 & 0.0677 & -0.0352 \\
${\text { Protein } \mathrm{R}^{2}}^{2}$ & 0.0043 & 0.1761 & $<0.0001$ & 0.1533 \\
\hline
\end{tabular}

${ }^{1}$ Null hypothesis: the slope of the line of best fit $=0$. Values $>0.05$ fail to reject this claim.

and Date was not significant $(P>0.05)$ during any of the 4 mo (Table 1).

The slopes of the lines of best fit for the protein test data during the months of December and July were significantly different from zero $(P<0.001)$ and had stronger coefficients of determination than any of the other plots in this study $\left(\mathrm{R}^{2}=0.20\right.$ and 0.35 for December and July, respectively). These results were consis-
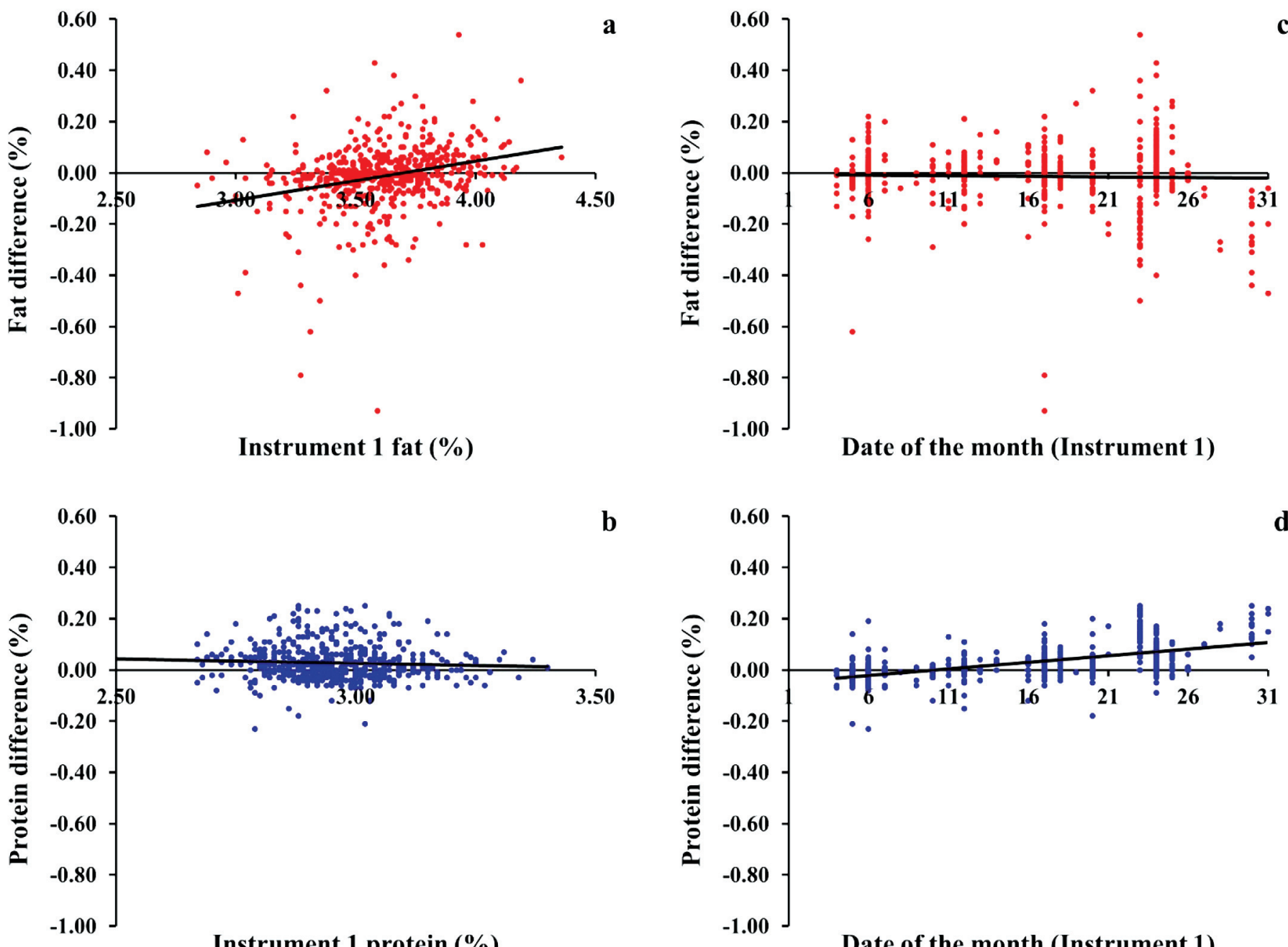

b

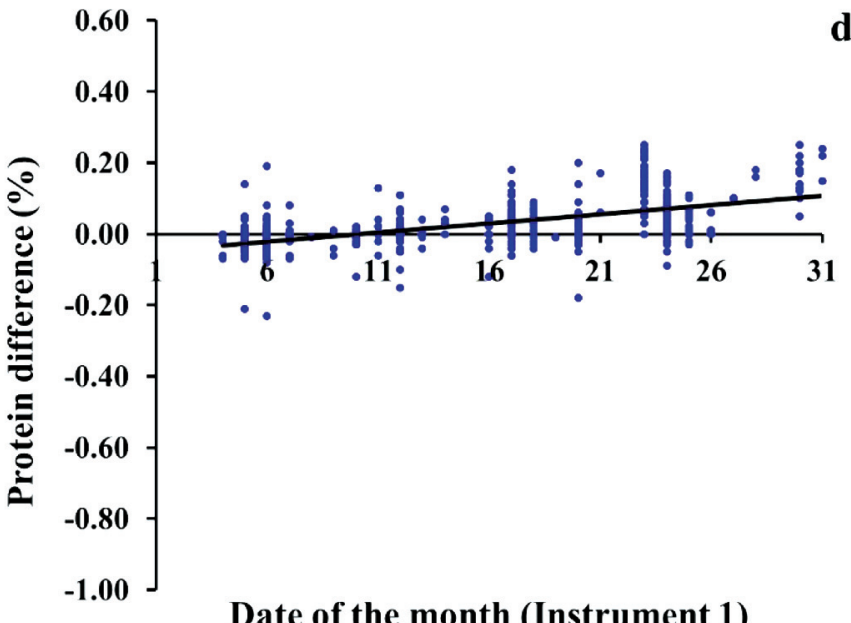

Figure 3. Linear regression graphs describing the correlations of (a) fat concentrations to differences in fat test values, (b) protein concentrations to differences in protein test values, (c) sampling dates of the month to differences in fat test values, and (d) sampling dates of the month to differences in protein test values between 2 mid-infrared spectrophotometers for the month of July 2011. Fat and protein differences are calculated as Instrument 1 test value minus Instrument 2 test value. Color version available online. 
Table 5. Linear regression parameters describing the effect of sampling date on the differences in fat and protein test values between 2 mid-infrared spectrophotometers for 4 mo during 2010 and 2011

\begin{tabular}{lcccr}
\hline Item & September 2010 & December 2010 & March 2011 & July 2011 \\
\hline Fat slope & $<0.0001$ & 0.0006 & -0.0012 & -0.0005 \\
Fat $P$-value & 0.9141 & 0.2076 & 0.0574 & 0.4687 \\
Fat $^{2}$ & $<0.0001$ & 0.0036 & 0.0080 & 0.0011 \\
Protein slope $^{2}$ & 0.0004 & -0.0020 & 0.0004 & 0.0051 \\
Protein $P$-value $^{1}$ & 0.1298 & $<0.0001$ & 0.0677 & $<0.0001$ \\
Protein R $^{2}$ & 0.0069 & 0.1994 & 0.0074 & 0.3464 \\
\hline
\end{tabular}

${ }^{1}$ Null hypothesis: the slope of the line of best fit $=0$. Values $>0.05$ fail to reject this claim.

tent with the protein test ANOVA (Table 2) in that the months of December and July both exhibited strong interaction effects $(P<0.001)$ between Instrument and Date. These results suggest that the 2 instruments' protein test values were drifting apart during the month of December and separately during the month of July, causing differences in protein determination between the 2 instruments to increase over time within month.

\section{Improving MIR Instrument Performance}

Mid-infrared milk analysis is a secondary testing method, and the slope and intercept for measurement of each component (e.g., fat, protein, or lactose) are adjusted using a set of reference milks. First, the analyst should ensure that the chemical reference methods used to establish the MIR calibration are appropriate for the analytes of concern. For milk fat, true protein, lactose, and total solids, the reference methods recognized in the United States are Mojonnier ether extraction (AOAC International, 2000; method 989.05; 33.2.26), Kjeldahl true protein (AOAC International, 2000; method $991.22 ; 33.2 .13)$, enzymatic anhydrous lactose (Lynch et al., 2007; method 2006.06), and forced-air oven drying for total solids (AOAC International, 2000; method 990.20; 33.2.44), respectively. Solids-not-fat (total solids minus fat) and other solids (solids-not-fat minus true protein) are calculated by difference.

An MIR instrument's mechanical and electrical components should also be examined routinely. This process is known as precalibration and Lynch et al. (2006) provide a detailed description of these procedures for MIR instruments used to test fluid milk samples. During precalibration, the instrument's flow system, homogenizer, repeatability on water, zero shift (cuvette condition), linearity, primary slope, repeatability on milk, purging efficiency, and intercorrection factors are inspected (Lynch et al., 2006). Proper selection of wavelengths for measurement of fat, protein, and lactose by transmission MIR spectrophotometry was discussed by Kaylegian et al. (2009a) and a set of optimized wavelengths and intercorrection factors determined. Use of these wavelengths will give excellent analytical performance when a Fourier transform MIR milk analyzer is set up properly.

If an instrument passes all precalibration testing, poor MIR analytical performance may be attributed to calibration set makeup. Kaylegian et al. (2006a) determined that creating calibration sets based on modified milk samples with orthogonal concentrations of fat, protein, and lactose produced more robust calibrations than producer milk calibration sets. These findings were attributed to the modified milk set's larger component concentration ranges, its more-even distribution of concentrations within those ranges, and its relative lack of collinearity among components of interest (i.e., fat and protein) compared with the producer milk sets. Calibration improvements were verified during a validation study (Kaylegian et al., 2006b), which indicated that using the modified milk set for calibration reduced fat, lactose, protein, and total solids reproducibility values (i.e., the differences between 2 MIR instruments measuring the same sample) by $46,52,61$, and $55 \%$, respectively.

Even though the population mean values for fat and protein for 2 instruments might not differ, the slope and intercept adjustments of these instruments may have been done using 2 different sources of reference samples. In this case, the mean values may agree but the slope over the range of concentration of fat and protein might be quite different, resulting in systematic over- or underpayment of producers who have milk fat or protein concentrations further away from the population mean. Although the proposed statistical method could identify this problem, these slope and intercept discrepancies can be minimized by calibrating MIR instruments using modified milk sets instead of producer milks (Kaylegian et al., 2006a).

\section{Proposed Implementation}

Due to the decreased sampling frequency and resource allocation that this approach could afford, its successful adoption could save handlers and regulatory laboratories expense while improving sustainability efforts. In order for this method to be implemented, 
further work should be done to verify that it performs well enough to identify MIR instruments that are out of tolerance for payment test performance, yet does not yield false positives on properly calibrated instruments. A validation study involving samples collected as described above being tested by multiple pairs of MIR instruments that are known to be performing well could be used to estimate specificity. A similar study involving pairs of correctly calibrated instruments and poorly calibrated instruments could be used to estimate sensitivity. These steps could be used to develop a consensus among regulatory laboratories regarding an $\alpha$ level at which action should be taken if the difference between 2 instruments exceeds the LSD. Additional studies that span an entire year's testing could also be done to evaluate the effect of seasonal differences in milk composition on MIR accuracy. Consideration should also be given to how adoption of a new statistical methodology might affect the industry's status quo regarding legal proceedings that rely on the validity of these results.

\section{CONCLUSIONS}

A multiple linear regression-based statistical approach to determine if 2 MIR instruments were producing different fat, protein, or other solids test values, on average, in a month was developed. This method could be implemented when split-sampling is not feasible. For 3 of the 4 mo examined, statistical differences in fat and protein determinations between the 2 instruments studied were established. Because the statistical model included factors for the effects of MIR instrument, milk producer, and date of milk collection, it was more sensitive at determining when instruments were performing differently from one another than was a $t$-test. Mean fat and protein LSD between instruments were 0.0126 and $0.0063 \%$ ( $\alpha=0.05)$, respectively, for the 4 mo studied. Increasing the confidence in this claim of difference will decrease the LSD. Regression analyses of the differences between the 2 instruments' fat or protein test values could be used to identify trends in MIR test data regarding the effects of composition or date of sample collection on instrument-to-instrument variation. The regression results in the present study agreed well with the multiple linear regression ANOVA findings.

\section{ACKNOWLEDGMENTS}

The authors thank the Testing Procedures Committee of the USDA Federal Milk Marketing Orders (Carrollton, TX) for partial funding of this research and the USDA Federal Milk Marketing Order 1 (Albany, NY) for supplying the raw data sets used for analysis.

\section{REFERENCES}

AOAC International. 2000. Official Methods of Analysis. 17th ed. AOAC Int., Gaithersburg, MD.

Barbano, D. M., and J. M. Lynch. 2006. Major advances in testing of dairy products: Milk component and dairy product attribute testing. J. Dairy Sci. 89:1189-1194.

Gilbert, G. R., G. L. Hargrove, and M. Kroger. 1973. Diurnal variations in milk yield, fat yield, milk fat percentage, and milk protein percentage of Holstein-Friesian cows. J. Dairy Sci. 56:409-410.

Glantz, S. A., and B. K. Slinker. 2001. Multicollinearity and what to do about it. Pages 185-187 in Primer of Applied Regression and Analysis of Variance. 2nd ed. McGraw-Hill, Inc., New York, NY.

Kaylegian, K. E., D. A. Dwyer, J. M. Lynch, D. E. Bauman, J. R. Fleming, and D. M. Barbano. 2009b. Impact of fatty acid composition on the accuracy of mid-infrared fat analysis of farm milks. J. Dairy Sci. 92:2502-2513.

Kaylegian, K. E., G. E. Houghton, J. M. Lynch, J. R. Fleming, and D. M. Barbano. 2006a. Calibration of infrared milk analyzers: Modified milk versus producer milk. J. Dairy Sci. 89:2817-2832.

Kaylegian, K. E., J. M. Lynch, J. R. Fleming, and D. M. Barbano. 2009a. Influence of fatty acid chain length and unsaturation on mid-infrared milk analysis. J. Dairy Sci. 92:2485-2501.

Kaylegian, K. E., J. M. Lynch, G. E. Houghton, J. R. Fleming, and D. M. Barbano. 2006b. Modified versus producer milk calibration: Mid-infrared analyzer performance validation. J. Dairy Sci. $89: 2833-2845$.

Lawson, J. 2010. Verifying assumptions of the linear model. Pages 27-30 in Design and Analysis of Experiments with SAS. CRC Press, Boca Raton, FL.

Lynch, J. M., D. M. Barbano, and J. R. Fleming. 2007. Determination of the lactose content of fluid milk by spectrophotometric enzymatic analysis using weight additions and path length adjustment: Collaborative study. J. AOAC Int. 90:196-216.

Lynch, J. M., D. M. Barbano, M. Schweisthal, and J. R. Fleming. 2006. Precalibration evaluation procedures for mid-infrared milk analyzers. J. Dairy Sci. 89:2761-2774.

Ott, R. L., and M. T. Longnecker. 2004. Linear regression and correlation. Page 528 in A First Course in Statistical Methods. Brooks/ Cole Thomson Learning Inc., Belmont, CA.

von Keyserlingk, M. A. G., N. P. Martin, E. Kebreab, K. F. Knowlton, R. J. Grant, M. Stephenson, C. J. Sniffen, J. P. Harner III, A. D. Wright, and S. I. Smith. 2013. Invited review: Sustainability of the US dairy industry. J. Dairy Sci. 96:5405-5425. 